\title{
Salivary flow rates, per se, may not serve as consistent predictors for dental caries
}

\author{
Cynthia P. Trajtenberg ${ }^{1}$, Juliana A. Barros ${ }^{2}$, Shalizeh A. Patel ${ }^{2}$, Leslie Miles $^{3}$, Charles F. Streckfus ${ }^{4 *}$ \\ ${ }^{1}$ Department of Periodontics, Case Western Reserve University School of Dental Medicine, Cleveland, USA \\ ${ }^{2}$ Department of Restorative Dentistry, Prosthodontics and Biomaterials, University of Texas School of Dentistry at Houston, Houston, \\ USA \\ ${ }^{3}$ Department of Medical/Surgical Rehabilitation, University of Texas School of Nursing at Houston, Houston, USA \\ ${ }^{4}$ Department of Diagnostic \& Biomedical Sciences, University of Texas School of Dentistry at Houston, Houston, USA \\ Email: ${ }^{*}$ charles.streckfus@uth.tmc.edu
}

Received 29 January 2013; revised 29 February 2013; accepted 8 April 2013

Copyright (C) 2013 Cynthia P. Trajtenberg et al. This is an open access article distributed under the Creative Commons Attribution License, which permits unrestricted use, distribution, and reproduction in any medium, provided the original work is properly cited.

\begin{abstract}
Background: This clinical study evaluated the effects of salivary flow rate, age, race, health status and medications on the incidence of caries. Methods: Caucasian and African-American men and women $(n=501)$, aged 22 - 93 years participated in the study. Stimulated (S) and unstimulated (U) parotid (P) and submandibular glands (SM) salivary secretions were collected. Stimulated whole saliva (SWS) was collected as control. Glandular stimulation was achieved using $2 \%$ citric acid at 30 -second intervals to the dorsal surface of the tongue. Salivary flow rates (SFR) were calculated by total weight of saliva divided by 5 minutes and expressed in $\mathrm{ml} /$ minute. Coronal caries were scored using the NIDR DMFS index. Carious lesions were classified according to tooth surfaces by a calibrated single examiner. Spearman correlation coefficients were calculated to determine the association between SFR with age and percentage of carious teeth. Multiple regression analyses were calculated at ( $p$ 0.05). Results: The variables gender, race, age, health status, medication usage and salivary function were not predictors for dental disease. Additionally, these risk factors were not risk factors for missing teeth. Conclusions: In conclusion, cross-sectional investigations are limited in their ability to identify the relevant variables for disease prediction. In addition, clinical and basic science investigations will be necessary to assess risk factors for dental caries.
\end{abstract}

Keywords: Salivary Glands; Saliva; Caries; Aging; Medications

\footnotetext{
"Corresponding author.
}

\section{INTRODUCTION}

Saliva has a myriad of functions which, when combined, plays a major role in maintaining a healthy oral environment $[1,2]$. In addition to providing local immunity within the oral cavity, the presence of specific salivary proteins and peptides provides the ability to sustain the tooth structure and buffer the by-products of bacteria [1, 2]. Furthermore, the secondary function of saliva is to cleanse the dentition and soft tissues of debris which provide a medium for the over production of destructive pathogens [1,2].

Xerostomia is a condition characterized by a severe decrease in salivary secretion [1-4]. There are many causes for hyposalivation including radiation therapy, chronic systemic diseases that accompany aging $[3,4]$ and a number of commonly used medications [5]. Reduced salivary function results in an unstable oral microenvironment whereby caries susceptibility is increased [1-5].

Dental caries is an infectious process where bacteria adhere to the tooth surface in a biofilm called dental plaque. Plaque is composed of salivary proteins that adhere to teeth, as well as bacteria and their byproducts. Plaque harbors the bacteria that initiate the demineralization process. Streptococcus mutans is one of several pathogens implicated with the caries process and is kept controlled by the presence of competing oral flora and salivary antimicrobial proteins [6]. Considering that saliva is implicated in the tooth remineralization process and protects the dentition from microbial assault, when its presence is compromised an increase in caries lesions can occur.

Dental caries is usually present among patient suffering from hyposalivation or xerostomia where oral microenvironment host immunity is altered [6-8]. Moreover, systemic disease coupled with their corresponding phar- 
macological treatment negatively impacts the innate and acquired immunity. With this in mind, this study was undertaken to determine the direct relationship between salivary gland function and caries experience in a welldefined patient population with and without systemic disease. For the purposes of this protocol, a generalized null hypothesis for the study will be stated as salivary flow is not a predictor of dental caries.

\section{SUBJECTS AND METHODS}

\subsection{Subjects}

The participants in this study were volunteers in the oral physiology component of the Baltimore Longitudinal Study of Aging (BLSA) and represented a selection of men and women ranging in age from 22 - 93 years [9]. In order to focus primarily on aging effects and to adjust for socioeconomic and educational variables, the study population was recruited primarily from the upper-middleclass segment of the population. They were educated with $71 \%$ having bachelors' degrees or higher and $85 \%$ of the participants was identified as having professional, technical and managerial occupations [9].

The BLSA participants were given thorough physical and psychological evaluations at their initial visit $[9,10]$. Health related subject data from their past and current medical history were used to characterize the systemic health status of the participants for this study [9]. The smoking behavior [11] and periodontal conditions of this cohort are described in depth (Albandar, 2000).

All participants were informed of their rights, the study procedures and were given an IRB-approved consent form. Each Individual was examined at one of four different time intervals: between 8:00 a.m. to 10:00 a.m., 11:00 a.m. to $12: 00$ a.m., 1:00 p.m. to 2:30 p.m. and 3:00 p.m. to 5:00 p.m.

\subsection{Inclusion/Exclusion Criteria}

The study consisted of the male and female subjects of varying racial backgrounds ranging in age from 18 to 100 years that were already enrolled as participants of the BLSA [9]. The criteria for entering the BLSA study are discussed in depth in prior publications [9,11]. All participants for this assessment had to have at least 10 teeth per maxillary and mandibular arches and be capable of signing the IRB consent form. Only edentulous and thos with less than 20 teeth were excluded from the protocol.

\subsection{Salivary Collections}

Unstimulated and stimulated secretions of the parotid and submandibular/sublingual glands were collected in this study. All unstimulated salivary secretions were first collected followed by the stimulated collections. The order of salivary collections was as follows: 1) unstimulated parotid gland saliva (UPGS), 2) unstimulated submandibular/sublingual gland saliva (USGS), 3) stimulated parotid gland saliva (SPGS), stimulated submandibular/sublingual gland saliva (SSGS) and stimulated whole saliva (SWS). It would have been of value to collect unstimulated whole for its physiological importance in nocturnal protection; however, only stimulated whole salivary secretions were collected due to the time constraint in the appointments. All collections were performed by one individual, all salivary samples are collected into pre-weighed plastic tubes, and the volume was determined gravimetrically. The procedures for each collection were as follows:

\subsubsection{Unstimulated Salivary Gland Collections}

UPGS was collected from the right parotid gland of all participants using the Carlson-Crittenden Cup. The Stenson's duct was isolated and the cup positioned. UPGS was collected for a one-minute equilibrium period followed by a five-minute period of actual total collection [12].

USGS were obtained using a $100 \mu$ micropipette or the NIDCR collector to collect fluid secreted by the Wharton's duct. These secretions usually enter the oral cavity through a common duct and can be obtained by first covering both Stenson's ducts of the parotid glands with gauze to prevent contamination of the sampling area. A cotton roll is placed in the vestibule adjacent to the lower lip, the floor of the mouth rinsed with water, and the Wharton's duct openings isolated with additional gauze. Samples were aspirated by light suction into the NIDCR collector [13].

\subsubsection{Stimulated Salivary Gland Collections}

SPGS was collected after the UPSG was obtained [10]. Stimulated saliva was collected for a two-minute equilibrium period followed by a two minute period of actual total collection. A $2 \%$ citric acid solution was applied to the dorsum of the tongue every 30 seconds during the two-minute collection and one minute equilibrium periods [12].

SSGS was collected in the same manner as the unstimulated samples except that the gustatory stimulus $(2 \%$ citric solution) was applied to the anterior dorsal surface of the tongue with a cotton swab at 30 -second intervals. Stimulated saliva was collected for a two-minute equilibrium period followed by a two-minute period of actual total collection [13].

SWS was collected using a standard piece of softened paraffin $(1.5 \mathrm{~g})$ placed in the participant's mouth. The patient is asked to swallow any accumulated saliva and is then instructed to chew the wax at a regular rate (45 
chews/min with a metronome). Three 2-minute samples are obtained. The volume and physical characteristics are recorded [14].

\subsection{Caries Diagnosis and Procedures}

The number of decayed, missing, filled tooth surfaces (DMFS) index was used in this study. The criteria included the sum of decayed, missing or filled permanent tooth surfaces and were used as a summary of cumulative caries experience. A single examiner evaluated the teeth and was well trained and calibrated with the diagnostic criteria. The examination procedures, scoring methodologies, and the guidelines for diagnosing coronal decay are defined in the Oral Health Surveys of the National Institute of Dental Research Diagnostic Criteria and Procedures Manual [15]. Radiological diagnostics were not used in this study.

Total number of teeth with current caries, restored and missing surfaces was recorded for each subject. Considering that there was a paucity of the total number of new carious surfaces among this population, it was combined with the number of filled and missing surfaces in order to formulate a total caries experience. Missing tooth surfaces that were lost due to trauma and orthodontic extractions were not included in the analyses. Third molars and root surfaces were not assessed.

\subsection{Statistical Analysis}

Statistical analyses were performed using SPSS ${ }^{\circledR}$ statistical software package [16]. Initially, descriptive analyses were conducted for each variable as well as cross-tabulations and graphical representations of the data. Chi Square and Fisher exact probability tests were used to analyze the dichotomous variables, while the Kendall's Tau was used to assess ordinal data. Spearman and Pearson correlations were also employed when appropriate.

Reduction on interval variables was employed using a factor analysis based on eigenvalues and total explained variance. For ordinal data, the Spearman Rho was used. After the variable reduction procedure, a fixed factorial model was constructed and analyzed using the general linear model procedure. Main effects were analyzed as well as any possible interactions that may occur between the independent variables. The analysis was performed using total affected surfaces as the dependent variable and salivary flow rates, age, race, health status and medication usage as the independent variables.

As active caries was rare among this cohort, the number of missing teeth and the number of sound teeth were dichotomized and logistic regressions were employed to determine risk factors for oral disease. Odds ratios and confidence intervals were calculated for each model.

\section{RESULTS}

The total population consisted of 501 ambulatory participants with a mean age of 58.6 and an age range of 22 93 years. The cohort with respect to age was near normal distribution with a skewness value of -0.10 and a kurtosis value of -0.93 . The panel was also nearly balanced with respect to gender with 256 men and 245 women participating in the study. The mean age for each gender was 61.5 and 55.6 respectively. Within the cohort, there were 449 Caucasians, 49 African-Americans and 2 Asians.

The participants that enrolled in this study were both medically healthy and medically compromised. Among these groups, 367 (73\%) participants in this study were determined as "healthy" by undergoing regular physical examination performed at the BLSA. Additionally, 78\% of the healthy participants were not taking any prescribed or over-the-counter medications during the course of the study while $22 \%$ were taking medications for other disorders (e.g., allergies, mild mental disorders). The balance of the participants were diagnosed with various medical disorders (128) such as hypertension (102), diabetes (7), past history of cancer (18) and a combination of hypertension with diabetes (1). Six participants were new entrants and were without confirmed medical histories. With the exception of those participants with a history of carcinoma, all were all taking prescription medications.

With respect to their dentition, the entire cohort exhibited a mean DMFS profile of $51.2( \pm 32.1)$ with $\mathrm{D}=0.1$ $( \pm 0.3), M=15.7( \pm 30.1)$ and $F=18.3( \pm 13.3)$ surfaces respectively (Table 1). There were a total of 132 individuals $(26 \%)$ that had a least one decayed or filled root surface with 76 being men and 56 being women. The number of affected root surfaces was too small in number to be further stratified and, therefore, did not warrant further statistical analyses. The women had approximately $13 \%$ more tooth surfaces than their male counterparts $(\mathrm{p}<0.01)$. Racially, Caucasians had more restored $(\mathrm{p}<0.05)$ and crowned surfaces $(\mathrm{p}<0.01)$ while the African American cohort had more sound surfaces $(\mathrm{p}<$ $0.01)$. There was also a moderate association between age and the total number of DMFS ( $\mathrm{r}=0.58$; $\mathrm{p}<0.001)$.

Medically, those diagnosed with medical illnesses exhibited significantly greater DMFS $(\mathrm{p}<0.001)$ than healthy individuals (Table 2). Likewise, those on prescription medications had significantly higher DMFS ( $\mathrm{p}<$ 0.001 ) as compared to the non-medicated group.

A total of 70 individuals (14\%) utilized removable prostheses. Twenty eight individuals $(6 \%)$ were totally edentulous while 30 participants utilized both maxillary and mandibular partial prostheses. Two participants had a complete upper and a lower partial denture. There were a total of 10 participants that no longer wore their lower partials due to their discomfort. 
Table 1. Means and standard deviations of decayed, missing, and restored surfaces according to gender and race.

\begin{tabular}{|c|c|c|c|c|c|c|c|c|}
\hline \multicolumn{2}{|c|}{ Independent Variables } & \multirow{2}{*}{$\begin{array}{c}\text { Statistics } \\
\text { Mean }\end{array}$} & \multirow{2}{*}{$\begin{array}{c}\text { DMFS } \\
54.0^{*}\end{array}$} & \multirow{2}{*}{$\begin{array}{c}\begin{array}{c}\text { Decayed } \\
\text { Surfaces }\end{array} \\
0.1\end{array}$} & \multirow{2}{*}{$\begin{array}{c}\text { Missing } \\
\text { Surfaces }\end{array}$} & \multirow{2}{*}{$\begin{array}{c}\begin{array}{c}\text { Filled } \\
\text { Surfaces }\end{array} \\
18.3\end{array}$} & \multirow{2}{*}{$\begin{array}{c}\begin{array}{c}\text { Sound } \\
\text { Surfaces }\end{array} \\
45.8\end{array}$} & \multirow{2}{*}{$\begin{array}{c}\begin{array}{c}\text { Crowned } \\
\text { Surfaces }\end{array} \\
18.3\end{array}$} \\
\hline & & & & & & & & \\
\hline & Men & $n$ & 256 & 256 & 256 & 256 & 256 & 256 \\
\hline & & Std. Dev. & 32.9 & 0.4 & 31.4 & 13.3 & 28.6 & 21.3 \\
\hline & & Mean & 48.3 & .04 & 14.1 & 17.5 & $52.6^{* *}$ & 16.7 \\
\hline \multirow[t]{10}{*}{ Gender } & Women & $n$ & 245 & 245 & 245 & 245 & 245 & 245 \\
\hline & & Std. Dev. & 31.1 & 0.2 & 28.6 & 12.4 & 29.5 & 18.9 \\
\hline & & Mean & 51.2 & 0.1 & 15.7 & 17.9 & 49.1 & 17.5 \\
\hline & Total & $n$ & 501 & 501 & 501 & 501 & 501 & 501 \\
\hline & & Std. Dev. & 32.1 & 0.3 & 30.1 & 12.8 & 29.2 & 20.2 \\
\hline & & Mean & $52.6^{* *}$ & 0.1 & 15.7 & $18.4^{*}$ & 47.9 & $18.4^{* * *}$ \\
\hline & Caucasian & $n$ & 450 & 450 & 450 & 450 & 450 & 450 \\
\hline & & Std. Dev. & 32.2 & 0.4 & 30.6 & 13.0 & 28.9 & 20.7 \\
\hline & & Mean & 38.2 & 0.04 & 15.2 & 14.2 & $59.9^{* *}$ & 8.8 \\
\hline & African American & $n$ & 49 & 49 & 49 & 49 & 49 & 49 \\
\hline \multirow[t]{7}{*}{ Race } & & Std. Dev. & 27.6 & 0.3 & 26.0 & 10.2 & 28.5 & 9.8 \\
\hline & & Mean & 57.0 & 0.0 & 28.0 & 0.5 & 59.0 & 28.5 \\
\hline & Asian & $n$ & 2 & 2 & 2 & 2 & 2 & 2 \\
\hline & Asian & Std. Dev. & 72.2 & 0.0 & 32.5 & 0.7 & 83.4 & 40.3 \\
\hline & & Mean & 51.2 & 0.1 & 15.7 & 17.9 & 49.1 & 17.5 \\
\hline & Total & $n$ & 501 & 501 & 501 & 501 & 501 & 501 \\
\hline & & Std. Dev. & 32.1 & 0.3 & 30.1 & 12.8 & 29.2 & 20.2 \\
\hline
\end{tabular}

Table 2. Means and standard deviations of DMFS for healthy and medically compromised patients.

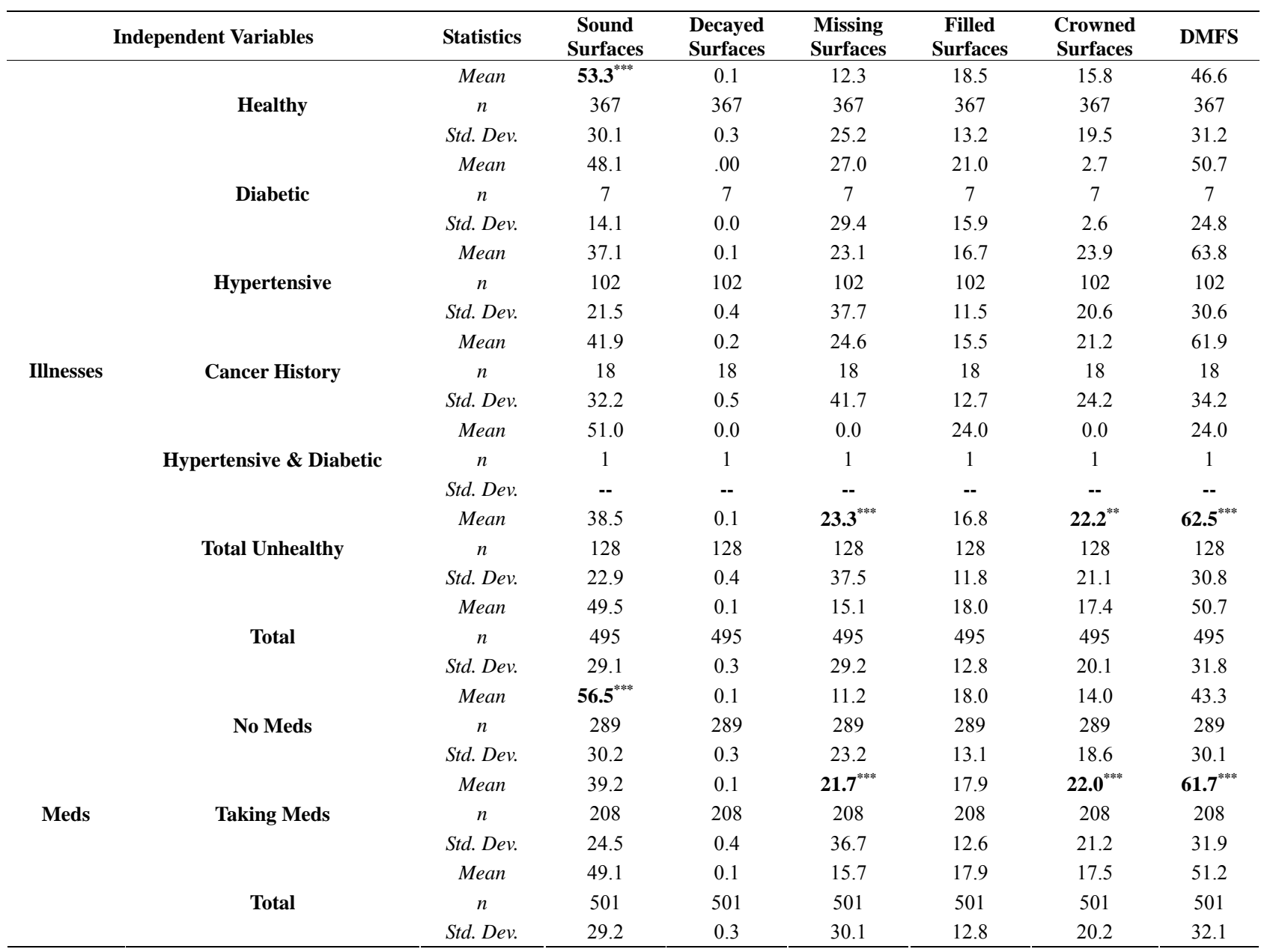


There were also significant results when the salivary flow rates were statistically examined (Table 3 ). The men had significantly high SWS flow rates $(\mathrm{p}<0.001)$, but lower SSG $(\mathrm{p}<0.001)$ salivary production when compared to the women. Additionally, African Americans had higher SSPG $(p<0.05)$, SSG $(p<0.05)$ and SWS $(p<0.001)$ flow rates. When examining the cohort for with respect to medical status, healthy participants had a higher SSPG $(\mathrm{p}<0.001)$, USSG $(\mathrm{p}<0.001)$ and SSG $(p<0.001)$ salivary flow when compared to those individuals diagnosed with medical illnesses. Those individuals not taking medications exhibited higher USPG $(p<0.05)$, SSPG $(p<0.001)$, USSG $(p<0.001)$ and SSG $(p<0.001)$ and SWS $(p<0.05)$ salivary flow rates as compared to the medicated group.

There were also numerous significant associations

Table 3. Analysis of variance for gender, race, health status and medications.

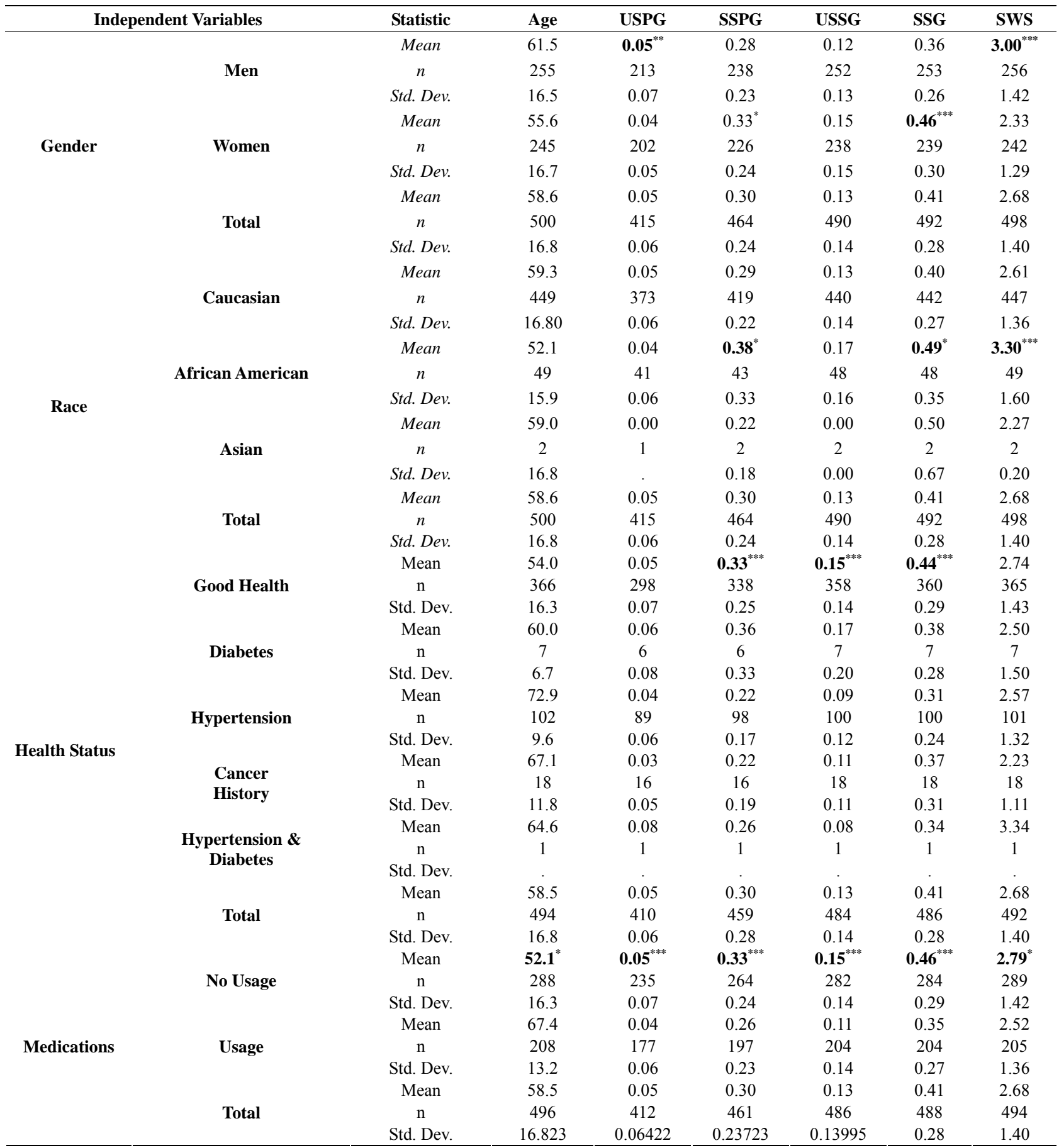


among the salivary gland flow rates, age, total affected surfaces, total missing teeth and total sound teeth; however, when partial correlations were performed controlling for age, there were no correlations among these factors. Individual regressions of the individual salivary flow rates with age indicate were significant at the $p<$ 0.001 levels; however, their $\mathrm{R}^{2}$ values were very low with SSG being the highest value at only $\mathrm{R}^{2}=0.12$. All regression slopes were negative with the steepest being
SSG at $\mathrm{m}=-0.006$. General linear modeling, which controlled for age also demonstrated that there was little association between the salivary flow rates and total affected surfaces.

Logistic regression analyses were performed using the number of missing teeth, the end stage for the dentition, as the dependent variable (Table 4). The variable was dichotomized into two separate groups. The non disease group consisted of 252 participants that retained their

Table 4. Logistic regression for gender, race, health status and medications with respect to flow rates.

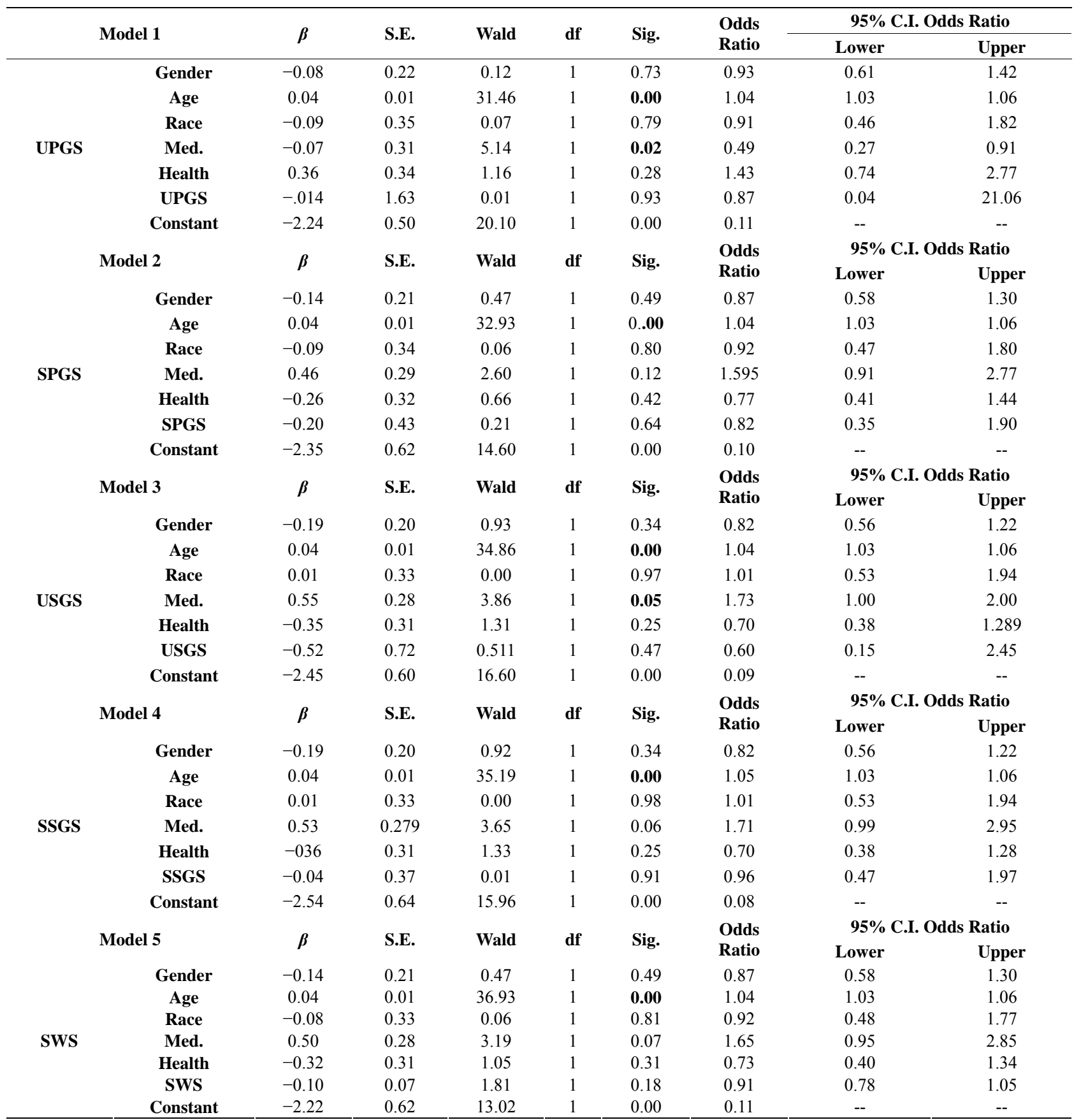

"Significant at the $\mathrm{p}<0.05 ;{ }^{* *}$ Significant at the $\mathrm{p}<0.01 ;{ }^{* * *}$ Significant at the $\mathrm{p}<0.001$. 
entire dentition and the diseased group consisted of 249 participants that had lost at least one tooth to either caries or periodontal disease. The dependent variables were gender, race, age, medication status, health status and the salivary flow rates. A model was performed for each salivary flow rate. Age and medication usage (UPGS, USGS) were significant at the $\mathrm{p}<0.001$ and $\mathrm{p}<0.05$ levels respectively; however, the odds ratio for both variables was very low. The salivary flow rates were not significant in these analyses. The Nagelkerke's $\mathrm{R}^{2}$ values for the models approximated 0.14 , which indicates a relatively poor association. Likewise, the -2 log likelihood values were extremely high indicating a poor association between the variables.

\section{DISCUSSION}

The data from the BLSA cohort was never designed to represent the entire US population, but was designed as an aging study controlling for socio-economic factors [9, 10,17]. Consequently it is difficult to make direct comparisons to the findings of the National surveys such as the Third National and Nutritional Examination Survey (NHANES III) which used unbiased population sampling techniques [18-20]; therefore, this panel will differ in the mean values for DMFS with respect to gender and raceethnicity as found in the NHANES III data.

Overall, the BLSA panel had only a slightly higher of mean number of permanent teeth when compared to the NHANES III data (BLSA 24.7 vs NHANES III 23.5). Unlike the NHANES III data, the men in this study had significantly higher DMFS than women; however, they had a significantly higher mean number of sound teeth as compared to the men [19].

Similarly, African Americans exhibited a significantly higher number of sound teeth as compared to the Caucasian cohort, which had a correspondingly significantly higher mean DMFS value (Table 1). This finding is supported by the study of Reid et al., which reported that when the "material factors" dental insurance, education, employment, poverty level and urban residence are comparable, that the risk for untreated caries is reduced by 21\% among African Americans [20]. The African American participants in this panel have "material factors" parity with their Caucasian counterparts in this study.

The subject's health status and corresponding medication usage had a dramatic impact on DMFS (Table 2). The healthy cohort had significantly more sound teeth ( $\mathrm{p}$ $<0.001$ ) while those that were unhealthy and or on medications exhibited higher DMFS values, but more importantly, twice as many missing surfaces. Those with a past history of a cancer experience had the highest mean DMFS values among the entire cohort.

With respect to the salivary function, there were also significant results when the salivary flow rates were statistically examined (Table 3). The men had significantly high SWS flow rates $(\mathrm{p}<0.001)$, but lower SSG $(\mathrm{p}<$ $0.001)$ salivary production when compared to the women. Additionally, African Americans had higher SSPG ( $\mathrm{p}<$ $0.05)$, SSG $(p<0.05)$ and SWS $(p<0.001)$ flow rates. There is very little in the literature concerning racial and gender effects on salivary function hence the authors can not explain these findings. With the exception of higher SSG among women, the majority of differences may be due to variations in salivary gland sizes [21]. Inoue et al. using magnetic resonance imaging, found an association between gland size, BMI, weight and salivary output [22]. Perhaps, this is responsible for the gender and racial differences between salivary gland flow rates. We can only speculate at this time.

When examining the results with respect to health, medical status and salivary function, the healthy participants had a higher mean SSPG ( $p<0.001)$, USSG ( $p<$ $0.001)$ and SSG $(p<0.001)$ salivary flow rate when compared to those individuals diagnosed with medical illnesses (Table 3). Those individuals not taking medications exhibited higher USPG $(\mathrm{p}<0.05)$, SSPG $(\mathrm{p}<$ $0.001)$, USSG $(\mathrm{p}<0.001)$ and SSG $(\mathrm{p}<0.001)$ and SWS $(p<0.05)$ salivary flow rates as compared to the medicated group. The unhealthy cohort is dominated by hypertensive participants. Hyposalivation among hypertensive individuals is well documented in the literature and may explain, in part, the lower mean DMFS and higher mean number of sound surfaces among the healthy cohort [3-5,22-24]. This effect couple with the loss of acinar cells due to aging may produce severe consequences for he hard tissues of the oral cavity [24-26].

Collectively the variables were assembled into an analytical regression models to determine if these variables could be used to predict dental disease (DMFS). When the model was controlled for age, there was no association with gender, race, health status, medication usage and salivary function with DMFS. Likewise as exhibited in Table 4, none of the variables in these models placed the individual at risk for tooth loss.

It is common knowledge that there is an increase in carious lesions among extreme cases where salivary flow rates are challenged such as in head and neck cancer radiation patients, Sjögrens sufferers and heavily medicated individuals there is a resultant increase in carious lesions among these populations. So why can't we accurately determine salivary flow rate cut values that indentify individuals at risk for increased caries prevalence? Ghezzi et al. suggest that it is due to a high rate of individual variability [27].Their results suggest that a $45 \%$ range in salivary flow rate variation and values below $45 \%$ of normal levels could be used to define salivary hypofunction [27]. Individual variability is, indeed, a part 
of the problem; however, we are suggesting that the current armamentarium lacks sufficient accuracy for determining true salivary flow rate cut points as it, by virtue of their design, introduce additional variables.

The Carlson-Crittenden Cup, for example, is a "onesize fits all" collection device that is used to collect secretions from Stenson ducts that significantly vary in size. Using clear plastic cup, you see in a number of cases where the duct maybe partially occluded due to the papilla filling nearly the entire inner portion of the annulus. In petite women, it is difficult to position the device over the Stenson papilla due to the close proximity of the zygomatic arch. The vacuum produced by rubber bulb, which holds the cup in position, may produce a valving effect on the Stenson's duct thereby artificially producing a false reduction in salivary flow rates. Even the stimulant may produce variability among the participants. Do the participants uniformly respond to the $2 \%$ citric solution? Will an aging decrease the subject's response to the intensity of the stimulant? If so, than this will affect the salivary flow rate.

The authors have as an example, focused on just one of the collection devices. Even the simplest methodology of collecting stimulating whole saliva yield moderate Intracorrelation (range $=0.54-0.79$ ) and Intercorrelation (range $=0.20-0.42$ ) coefficients and varied according to the time of day [28]. As a consequence, cross-sectional investigations are limited in their ability to identify relevant salivary flow rates for disease prediction [29]. We simply cannot control for temporal relationships and cohort effects. The lack of accuracy among the non-invasive collecting devices may also contribute to the lack of success in demonstrating when a subject is at risk for caries. Further, clinical and basic science investigations will be necessary to assess risk factors for dental disease.

\section{CONCLUSION}

The risk of caries and its relationship to salivary function is a complex and multifactorial phenomenon. In extreme situations such as those experiencing head and neck radiation when salivary flow is severely diminished, we observe an elevated caries experience; however, when there is a chronic diminution of salivary spanning a lifetime, the end results may not as abrupt or as observed in head and neck radiation. As experienced in this cohort, a low flow rate did not necessitate an elevated caries experience. It may be possible that the individual has adapted to its situation. Due to individual variability, the imprecision of non-invasive salivary gland assessment techniques, host response to microbial assault and numerous other confounding variables, we may not be able to determine a "cut-point" as to where salivary hypofunction initiates a decrease in oral health. The clinical significance of this report is that it isn't the salivary flow rates per se that are causing the increases in the caries experience but rather the critical lack of its constituents which upsets the homeostasis of the oral ecology.

\section{REFERENCES}

[1] Dowd, F.J. (1999) Saliva and dental caries. Dental Clinics North of America, 43, 579-597.

[2] Mandel, I. (1989) The role of saliva in maintaining oral homeostasis. Journal American Dental Association, 119 , 298-304.

[3] Streckfus, C.F. (1995) Salivary function and hypertension. Journal American Dental Association, 126, 1012-1017.

[4] Streckfus, C.F., Wu, A., Ship, J. and Brown, L.J. (1994) A comparison of stimulated parotid salivary flow rates in elderly normotensive, hypertensive, and controlled hypertensive individuals taking hydrochlorothiazide antihypertensive medications. Oral Surgery Oral Medicine Oral Pathology Oral Radiology Oral Endodontology, 23, 280283.

[5] Sreebny, L.M. and Schwartz, S.S. (1986) A reference guide to drugs and dry mouth. Gerodontology, 5, 75-99. doi:10.1111/j.1741-2358.1986.tb00055.x

[6] Selwitz, R.H., Ismail, A.I. and Pitts, N.B. (2007) Dental caries. Lancet, 369, 51-59. doi:10.1016/S0140-6736(07)60031-2

[7] Scheie, A. and Peterson, F. (2004) The biofilm concept: Consequences for future prophylaxis of oral diseases? Critical Reviews of Oral Biology and Medicine, 15, 4-12. doi:10.1177/154411130401500102

[8] Papas, A.S., Joshi, A. and MacDonald, S.L., MaravelisSplagounias, L., Pretara-Spanedda, P. and Curro, F.A. (1993) Caries prevalence in xerostomic individuals. Journal of the Canadian Dental Association, 59, 171-179.

[9] Shock, N.W., Greulich, R.C., Andres, R., et al. (1984) Normal human aging: The Baltimore longitudinal study of aging. US Government Printing Office, Washington DC.

[10] Baum, B.J. (1981) Characteristics of participants in the oral physiology component of the Baltimore longitudinal study of aging. Community Dental Oral Epidemiology, 9, 128-134. doi:10.1111/j.1600-0528.1981.tb01043.x

[11] Albandar, J.M., Streckfus, C.F., Adesanya, M.R. and Winn, D.A. (2000) Cigar, pipe and cigarette smoking as risk factors for periodontal disease and tooth loss. Journal of Periodontology, 71, 1874-1881. doi:10.1902/jop.2000.71.12.1874

[12] Heft, M.W. and Baum, B.J. (1984) Unstimulated and stimulated parotid salivary flow rate in different age groups. Journal of Dental Research, 63, 1182-1185. doi:10.1177/00220345840630100101

[13] Tylenda, C.A., Ship, J., Fox, P.C. and Baum, B.J. (1988) Evaluation of submandibular salivary flow rates in different age groups. Journal of Dental Research, 67, 225-228. doi:10.1177/00220345880670091501

[14] Navasesh, M. and Christensen, C. (1982) A comparison of whole mouth resting and stimulated salivary measure- 
ments. Journal of Dental Research, 61, 1158-1162. doi: $10.1177 / 00220345820610100901$

[15] Oral Health Surveys of the National Institute of Dental Research (1991) Diagnostic criteria and procedures. N.I.H. Publication No. 91-2870.

[16] Norušis, M.J. (2000) SPSS ${ }^{\circledR} 12.0$ guide to data analysis. Prentice Hall, Upper Saddle River.

[17] Copeland, L., Krall, E.A., Brown, L.J., Garcia, R.I. and Streckfus, C.F. (2004) Predictors of tooth loss between two adult populations. Journal of Public Health Dentistry, 64, 31-37. doi:10.1111/j.1752-7325.2004.tb02723.x

[18] Brown, L.J., Winn, D.M. and White, B.A. (1996) Dental caries, restorations and tooth conditions in US Adults, 1988-1991. Journal American Dental Association, 127, 1315-1325.

[19] Winn, D.M., Brunelle, J.A., Selwitz, R.H., Kaste, L.M., Oldakowski, R.J., Kingman, A. and Brown, L.J. (1996) Coronal and root caries in the dentition of adults in the United States, 1988-1991. Journal of Dental Research, 75, 642-651.

[20] Reid, B.C., Hyman, J.J. and Macek, M.D. (2004) Race/ ethnicity and untreated dental caries: The impact of material and behavioral factors. Community Dental Oral Epidemiology, 32, 329-336. doi:10.1111/j.1600-0528.2004.00165.x

[21] Navazesh, M., Brightman, V.J. and Pogoda, J.M. (1996) Relationship of medical status, medications, and salivary flow rates in adults of different ages. Oral Surgery Oral Medicine Oral Pathology Oral Radiology Oral Endodontology, 81, 172-176. doi:10.1016/S1079-2104(96)80410-0

[22] Inoue, H., Ono, K., Masuda, W., Morimoto, Y., Tanaka,
T., Yokota, M. and Inenaga, K. (2006) Gender differences in unstimulated whole saliva flow rate and salivary gland sizes. Archives of Oral Biology, 51, 1055-1060. doi:10.1016/j.archoralbio.2006.06.010

[23] Bahn, S.L. (1972) Drug related dental destruction. Oral Surgery Oral Medicine Oral Pathology Oral Radiology Oral Endodontology, 33, 49-54.

[24] Scott, J. (1986) Structure and function in aging human salivary glands. Gerodontology, 5, 149-158. doi:10.1111/j.1741-2358.1986.tb00062.x

[25] Scott, J. (1977) Quantitative age changes in the histological structure of human submandibular salivary glands. Archives of Oral Biology, 22, 221-227. doi:10.1016/0003-9969(77)90158-3

[26] Scott, J. (1979) The proportional volume of mucous acinar cells in normal human submandibular salivary glands. Archives of Oral Biology, 24, 479-481. doi:10.1016/0003-9969(79)90012-8

[27] Ghezzi, E.M., Lange, L.A. and Ship, J.A. (2000) Determination of variation of stimulated salivary flow rates. Journal of Dental Research, 79, 1874-1878. doi:10.1177/00220345000790111001

[28] Streckfus, C.F., Bigler, L., Dellinger, T.D., Dai, X., Cox, W.J., McArthur, A., Kingman, A. and Thigpen, J.T. (2001) Reliability assessment of soluble c-erbB-2 concentrations in the saliva of healthy women and men. Oral Surgery Oral Medicine Oral Pathology Oral Radiology Oral Endodontology, 91, 174-180. doi:10.1067/moe.2001.111758

[29] Ship, J.A., Fox, P.C. and Baum, B.J. (1991) How much saliva is enough? "Normal" function defined. Journal of American Dental Association, 122, 63-69. 